\title{
Review of the State of Acoustic Telemetry and Constraining the Adaptation Dimension for Acoustic Telemetry Systems
}

\author{
Arthur B. Baggeroer \\ Massachusetts Institute of Technology \\ Cambridge, MA 02139 \\ phone: (617) 253-4336, email: $\underline{\text { abb@arctic.mit.edu }}$ \\ Award N000149710796 \\ http://acoustics.mit.edu
}

\section{LONG-TERM GOALS}

The effort has two long term goals i) assess the state of the art of acoustic telemetry systems including demonstrated experimental performance and ii) improve the performance of telemetry systems by constraining adaptation dimensions. First, the review activity aims to provide both funding managers and researchers alike an unbiased assessment of what the underwater acoustic telemetry community has accomplished. By drafting such a comprehensive research map, discussions regarding future research directions may focus on clearly identified performance deficiencies. As an example, many existing telemetry systems either neglect or only grossly account for the underlying physical mechanisms that define the channel. A prominent theme of the review is that a more explicit incorporation of channel models in the design of receivers will yield improved performance. Posing and supporting assertions such as this is the fundamental objective of the review. The second set of research objectives is centered on controlling the degrees of freedom implemented in underwater acoustic telemetry receivers. Improved tap definition and placement should lead to increased levels of intersymbol interference suppression, higher channel tracking rates, and improved numerical stability in the equalizer algorithms.

\section{OBJECTIVES}

I will ascertain the state of the art in underwater acoustic telemetry to include demonstrated performance and current research trends. A comparative analysis of published work by the different groups in the community will form the foundation for a set of recommendations for future work. I will determine the level of progress in specific technology areas such as underwater networks, coding methodologies, and receiver signal processing. For the second thrust of this work, I will establish how the time variability and spatial structure of the underwater acoustic channel may be efficiently represented, as well as exploited, in both receiver and transmitter subsystem of a complete acoustic modem. Discrete doppler effects induced by platform motion may be effectively mitigated by modelbased receiver structures. The spatial character of the channel may be adaptively estimated and incorporated into both modulation strategies and the specific architecture of space-time adaptive processing algorithms.

\section{APPROACH}

The success of the review hinges on assembling a comprehensive and accurate database of previous and ongoing underwater acoustic telemetry projects. A large component of the database has come, and continues to come, from an exhaustive literature survey including both peer-reviewed journals and 


\section{Report Documentation Page}

Form Approved

OMB No. 0704-0188

Public reporting burden for the collection of information is estimated to average 1 hour per response, including the time for reviewing instructions, searching existing data sources, gathering and maintaining the data needed, and completing and reviewing the collection of information. Send comments regarding this burden estimate or any other aspect of this collection of information,

including suggestions for reducing this burden, to Washington Headquarters Services, Directorate for Information Operations and Reports, 1215 Jefferson Davis Highway, Suite 1204, Arlington

VA 22202-4302. Respondents should be aware that notwithstanding any other provision of law, no person shall be subject to a penalty for failing to comply with a collection of information if it

does not display a currently valid OMB control number.

1. REPORT DATE

30 SEP 1999

4. TITLE AND SUBTITLE

Review of the State of Acoustic Telemetry and Constraining the Adaptation Dimension for Acoustic Telemetry Systems

6. AUTHOR(S)

7. PERFORMING ORGANIZATION NAME(S) AND ADDRESS(ES)

Massachusetts Institute of Technology,77 Massachusetts

Avenue,Cambridge,MA,02139

9. SPONSORING/MONITORING AGENCY NAME(S) AND ADDRESS(ES)

\section{DATES COVERED}

00-00-1999 to 00-00-1999

5a. CONTRACT NUMBER

5b. GRANT NUMBER

5c. PROGRAM ELEMENT NUMBER

5d. PROJECT NUMBER

5e. TASK NUMBER

5f. WORK UNIT NUMBER

8. PERFORMING ORGANIZATION REPORT NUMBER

10. SPONSOR/MONITOR'S ACRONYM(S)

11. SPONSOR/MONITOR'S REPORT NUMBER(S)

12. DISTRIBUTION/AVAILABILITY STATEMENT

Approved for public release; distribution unlimited

13. SUPPLEMENTARY NOTES

14. ABSTRACT

15. SUBJECT TERMS

16. SECURITY CLASSIFICATION OF:

a. REPORT

unclassified b. ABSTRACT

unclassified c. THIS PAGE

unclassified
17. LIMITATION OF ABSTRACT

Same as

Report (SAR)
18. NUMBER 19a. NAME OF

OF PAGES RESPONSIBLE PERSON

5 
conference proceedings. Both I and my research assistant for this work, Mr. Daniel Kilfoyle, have extensive connections with the Woods Hole Oceanographic Institution as well as other organizations actively involved in this area such as the Naval Undersea Warfare Center (NUWC), Northeastern University, and the Space and Naval Warfare Systems Command (SPAWAR) which provide additional avenues for gathering information. Participation in focused review meetings provides access to unpublished research.

Efficient accommodation of underwater acoustic channels with discrete doppler components was addressed by Dr. Trym Eggen in his dissertation work. He combined a channel model with a linear equalizer. The channel model utilized a Kalman filter to recursively estimate a discrete multi-tone Doppler characterization of the channel. The intent was to reduce the tracking burden on the equalizer tap weights. Mr. Daniel Kilfoyle is using a unique decomposition of the underwater channel as described by a frequency dependent modulation function to design modulation and demodulation filters for spatial taps that will result in higher throughputs and reliability for telemetry systems. The work will be experimentally validated.

\section{WORK COMPLETED}

An electronic database of approximately 140 journal articles and conference proceedings has been assembled including a short, critical review of each by Mr. Kilfoyle. Preliminary state of the art summaries have been presented at several public forums including the June, 1998 meeting of the Acoustical Society of America in Seattle, Washington, (Baggeroer, 1998) and several ONR workshops. The review has now been finalized, reviewed, and accepted by the IEEE Journal of Oceanic Engineering for publication in the January, 2000 special issue on underwater acoustic communication. Pre-press versions have been transmitted to several ONR officials.

Dr. Trym Eggen completed the development and evaluation of the multi-doppler tone coherent receiver. This is documented in his Ph.D. thesis (Eggen, 1997) as well as a paper submitted to the IEEE Journal of Oceanic Engineering.

Mr. Kilfoyle has experimentally demonstrated the viability of simultaneously and independently exciting multiple underwater communication channels in a shallow water environment using both a source transducer array and a receiving hydrophone array.

\section{RESULTS}

While the actual accomplishments of the underwater acoustic telemetry community have been clearly identified in the ONR sponsored review (Kilfoyle and Baggeroer, in press), another important result of this year's work has been the progress seen in addressing the recommendations presented in last years annual report.

- Bridge the gap between the ocean modeling community and the signal processing community in order to spur development of modeling tools suitable for the frequencies and bandwidths of telemetry signals. The ongoing planning for a high-frequency acoustic communication and channel characterization experiment sponsored by the ONR Ocean Acoustics Division begins to address this point. 
- Receiver architecture improvements should grow out of more insight into the physical ocean channel as characterized by the classical, potentially time-varying, scattering function. This point is gaining acceptance among ONR sponsored acoustic communication researchers.

- A suite of benchmark channels would be of great utility to all members of the underwater acoustic telemetry community and should be generated, initially from accurate data sets and eventually from suitable models. No progress has been made in this area.

The potential for increased data rates from existing AUV platforms and future fixed node networks was experimentally demonstrated last year by Mr. Kilfoyle and presented at the Acoustical Society of America conference in Berlin, Germany (March, 1999.) Having shown theoretically that the ability to simultaneously and independently excite multiple, spatially distinct communication channels in the ocean leads to performance improvements equivalent to $3-6 \mathrm{~dB}$ improvements in signal to noise ratio (SNR), Mr. Kilfoyle experimentally demonstrated that capability in 15 meter water in Buzzards Bay, MA. Using a six element transducer array and a 16 element receiver array, $4 \mathrm{kHz}$ bandwidth acoustic signals on a $9.6 \mathrm{kHz}$ carrier coherently conveyed information using 2-level, 4-level, and 16-level constellations. In one test, two signals were alternately sent. The first waveform had all 6 transducers convey a single binary phase shift keyed (BPSK) packet. The second waveform employed the top 3 transducers to send one BPSK packet while the bottom 3 transducers simultaneously sent another. Total average transmitted power was held constant during the test. Each packet type was sent approximately 160 times. The histogram in figure 1 shows the number of packets of each type that had an output SNR in $2 \mathrm{~dB}$ wide bins between $4 \mathrm{~dB}$ and $20 \mathrm{~dB}$. The packets simultaneously sent over the subarrays suffered an average $4 \mathrm{~dB}$ drop in output SNR compared to the single data stream packets. Since this error increase was only modestly greater than what one would attribute to the $3 \mathrm{~dB}$ reduction in power per information bit implies, we conclude the expected performance improvements were achieved. 


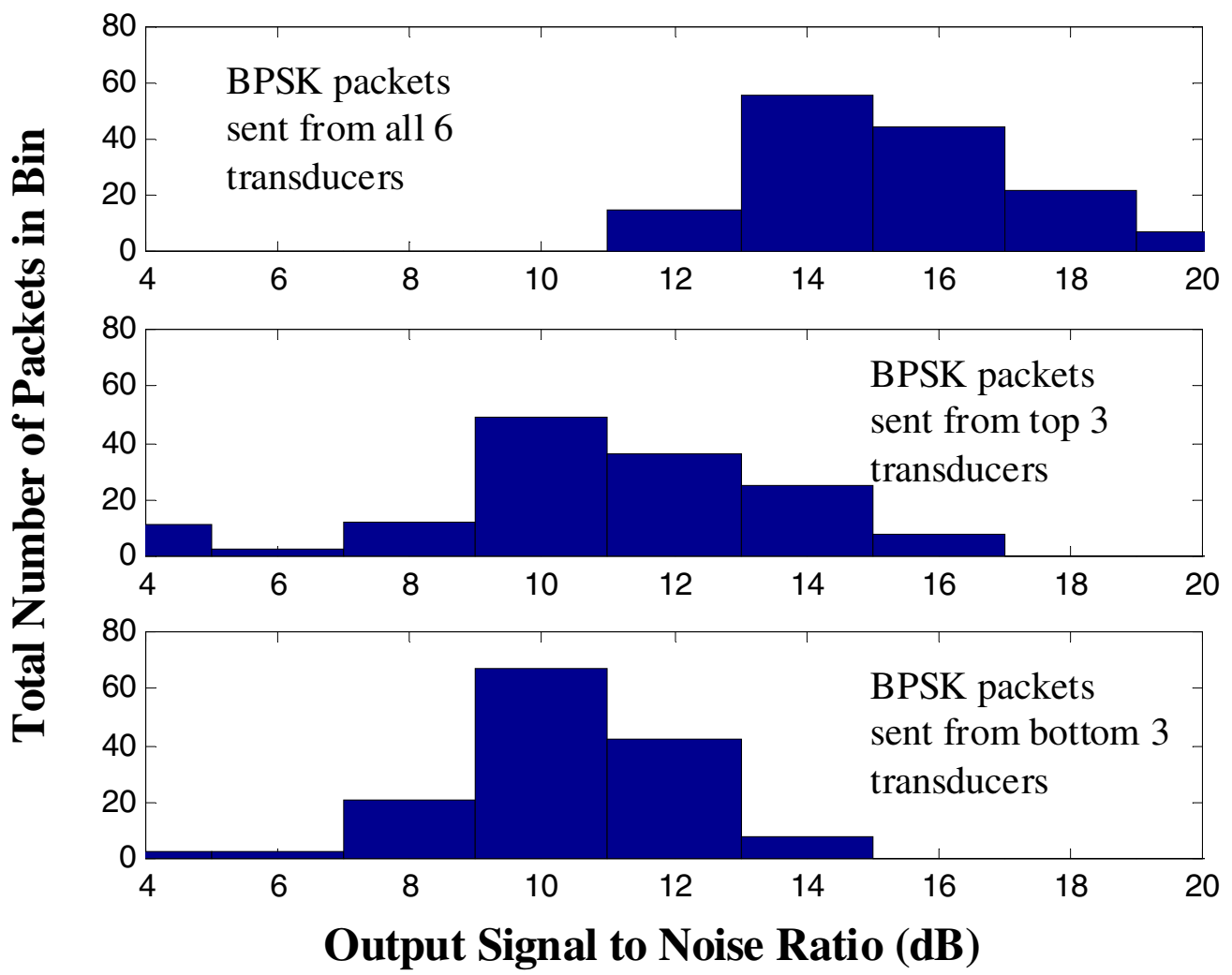

1. Summary of acoustic communication performance comparing conventional signaling (sending the same packet from all transducers) to spatially modulated signaling (simultaneously sending two packets with distinct spatial signatures) with an average power constraint. Total number of received packets having the designated output SNR are shown in the histogram above. The performance nearly achieves what is expected over additive white Gaussian noise parallel channels.

\section{IMPACT/APPLICATION}

The state of the art summary and recommendations that arise from the published review should serve to guide strategic discussions of future work, specifically that funded by ONR. The work on management of degrees of freedom will lead to more robust, higher performance coherent modems for use by the U.S. Navy and oceanographic community.

\section{TRANSITIONS}

The results of the review continue to be disseminated in professional forums as well as ONR workshops. The results have also been discussed with ASTO and the review has been presented to OPNAV-92. The multi-tone Doppler receiver and spatial decomposition approaches have yet to be used in conjunction with other modem development efforts. 


\section{RELATED WORK}

There has been extensive participation in the ongoing planning process for a sequence of high frequency acoustic communication experiments managed by the ONR Ocean Acoustics Branch. A principal objective of those tests is to reconcile communication performance with concurrent environmental measurements. Mr. Kilfoyle was involved in the 1998 planning conference while Prof. Baggeroer helped guide this year's planning conference.

\section{PUBLICATIONS}

Baggeroer, Arthur and Daniel Kilfoyle, "Telemetry and the Underwater Channel: Progress and Challenges," Proceedings of the 135th Meeting of the Acoustical Society of America, June 20-26, 1998.

Eggen, Trym, “Underwater Acoustic Communication over Doppler Spread Channels,” Ph.D. dissertation, Massachusetts Institute of Technology, June, 1997.

Kilfoyle, Daniel and Arthur Baggeroer, "The State of the Art in Underwater Acoustic Telemetry," IEEE Journal of Oceanic Engineering, in press. 